\title{
Review Article \\ From Local Adaptation to Speciation: Specialization and Reinforcement
}

\author{
Thomas Lenormand \\ CEFE-UMR 5175, 1919 Route de Mende, 34293 Montpellier Cedex 5, France \\ Correspondence should be addressed to Thomas Lenormand, thomas.lenormand@cefe.cnrs.fr
}

Received 29 July 2011; Revised 21 November 2011; Accepted 12 December 2011

Academic Editor: Marianne Elias

Copyright ( $) 2012$ Thomas Lenormand. This is an open access article distributed under the Creative Commons Attribution License, which permits unrestricted use, distribution, and reproduction in any medium, provided the original work is properly cited.

Local adaptation is the first step in the process of ecological speciation. It is, however, an unstable and dynamic situation. It can be strengthened by the occurrence of alleles more specialized to the different habitats or vanish if generalist alleles arise by mutations and increase in frequency. This process can have complicated dynamics as specialist alleles may be much more common and may maintain local adaptation for a long time. Thus, even in the absence of an absolute fitness tradeoff between habitats, local adaptation may persist a long time before vanishing. Furthermore, several feedback loops can help to maintain it (the reinforcement, demographic, and recombination loops). This reinforcement can occur by modifying one of the three fundamental steps in a sexual life cycle (dispersal, syngamy, meiosis), which promotes genetic clustering by causing specific genetic associations. Distinguishing these mechanisms complements the one- versus two-allele classification. Overall, the relative rates of the two processes (specialization and reinforcement) dictate whether ecological speciation will occur.

\section{Introduction}

The debate over speciation is not new [1-7] and is a complex subject as speciation represents a "cluster of theories woven from many strands" [7] that can be approached from different angles: sympatry versus allopatry, intrinsic versus extrinsic selection, premating versus postmating isolation, isolation versus adaptation, one-allele versus two-allele mechanisms, primary versus secondary contact, genic versus genomic, and so forth. This diversity of possible approaches can even lead to a synthesis based on the idea of a menu with different possible options for each course [8].

One view has reemphasized Darwin's view that speciation was about adaptation to different habitats or niches $[2,4,9,10]$. At a microevolutionary scale, this process often starts within species as local adaptation. This can be the beginning of future divergence and eventually speciation. However, the basic process of local adaptation is often seen as too "preliminary" to stand at the core of a theory for speciation. Indeed, "mere differential adaptation (...) does not constitute species" [1] and hybrid unfitness is often the main selective scenario envisioned to be necessary. Hybrid unfitness can be caused by alternative adaptive peaks in the same habitat, whereas local adaptation usually occurs with a single peak that differs in different habitats, which is quite different, even if not always recognized as such $[1,8,11]$. Of course, both phenomenon are not exclusive and evaluating their relative importance is often controversial [12], as both produce indistinguishable spatial patterns [13]. We will see that the route from local adaptation to speciation has been explored in a somewhat separate theoretical corpus that still has to be fully incorporated to a microevolutionary view of speciation.

Environmental heterogeneity is pervasive and local adaptation is the direct selective consequence of different selection pressures occurring in different places [14]. Local adaptation - the greater average fitness of local individuals compared to immigrants-occurs whenever the "grain" of habitat is sufficiently coarse compared to the scale of dispersal $[15,16]$. It has various consequences ranging from niche evolution, evolution of specialization, to ecological speciation. In the context of speciation, local adaptation is a selective context that is universal: it applies to sexual or asexual species alike, contrary to selection pressures caused 
by hybrid incompatibilities. However, it also has specific features. First it is a dynamic and gradual process. Local adaptation can constantly strengthen if new alleles better adapted to local conditions arise and increase in frequency, or disappear if generalist genotypes spread or if differentiation is swamped by gene flow. In contrast, a situation of secondary contact is not a gradual process, but often the abrupt exposure of genetic incompatibilities that have accumulated independently in allopatry. It results in tension zones that can be stable over long periods of time [17]. Second, hybrids between two locally adapted parental genotypes are often not the worst genotype in any of the environments. With local adaptation, there is not selection against hybrids per se: there is selection against alleles or genotypes that are not locally favorable. This distinction has important consequences understanding how reinforcement works in the context of local adaptation compared to the case of reinforcement to avoid producing unfit hybrids. Here, I use reinforcement sensu lato to mean the evolution of premating isolation resulting from selection against hybrids or locally maladapted genotypes [18]. In this paper, I will focus on these two topics and try to show that their outcomes are often less straightforward than early models have suggested, and that the theory of local adaptation has to be more fully integrated into a global view of speciation and diversification.

\section{Topic 1-The Dynamics of Local Adaptation}

Because niche specialization can occur in the presence of gene flow in primary contact and does not require time in isolation to evolve genetic incompatibilities, it results in a more dynamical process, where further niche specialization may or may not occur, with direct consequences on reinforcement towards more isolation.

2.1. Habitats. Models of local adaptation and ecological speciation most often start by assuming that there are different habitats exchanging migrants (with the rate of migration corresponding to the cases of sympatry, parapatry and allopatry [3]). However, the definition of habitat can be problematic. Field ecologists would certainly define it by a combination of biotic/abiotic conditions in a landscape. For instance, when thinking about land plants, they would categorize soil type, moisture, temperature, light, slope, disturbance regime, and so forth. From an ecological genetics perspective, a first difficulty is to define these variations at the relevant scale, that is, at the scale of dispersal of the focal species $[15,16]$. A second difficulty is to account for distance. Because dispersal is most often distance limited, the spatial configuration makes a difference in terms of habitat definition. This is well known in complex or mosaic habitats [19], but is true even in very simple landscapes [20]. A given ecological condition will be less prone to local adaptation if close to a habitat boundary than if surrounded by identical conditions. Boundaries may even favor the local evolution of generalists, something that contradicts the simple competition exclusion principle [20]. Finally, conditions also vary through time, which strongly limit the scope for the long term maintenance of locally specialized genotypes. Overall, locally adapted genotypes are unlikely to arise "on every bush" [21], yet are expected to arise frequently.

2.2. Local Adaptation and the Origin of Tradeoffs. One basic process by which local adaptation arises is when, within a large enough habitat, an allele increases in frequency that is beneficial inside but deleterious/neutral outside this habitat. The condition for this increase in frequency is determined by the strength of selection inside and outside, the size of the pocket relative to the scale of dispersal and by possible gene flow asymmetries between habitats $[15,22]$. Any allele with a sufficient benefit inside will increase in frequency no matter if it presents strong deleterious effects outside. This process does not necessarily favor alleles exhibiting little antagonistic effect across habitats; anything goes that is sufficiently favorable locally. At the same locus, allele replacement can occur in both directions, favoring either stronger or weaker specialization [23]. Another possibility is that neutral mutations drift at high frequency locally despite being deleterious elsewhere [14, 24, 25]. However, even if this can occur [26], it requires very limited gene flow and may be globally less conducive to strong local adaptation.

This process has no reason to stop and lead to a process of "amelioration" [27] whereby new favorable alleles replace previous ones at a given locus [23, 28-30], modifier alleles at new loci evolve to correct for deleterious side effects of previous ones [31-33], duplications and new functions can arise [34-36], and so forth. The question is whether this amelioration will lead to the evolution of generalist genotypes that can accommodate all habitats or whether local adaptation will strengthen and lead to specialized ecotypes that have diverged at a large number of loci. The answer is not straightforward. The first approach is to build a model imposing a trade-off curve between habitats. Whether specialists or generalists evolve depends then on the shape of this trade-off curve $[37,38]$. Globally speaking, more concave curves facilitates the evolution of specialists, whereas more convex ones favor generalists, and sometimes both can coexist $[37,38]$. However, there is no clear ultimate reason for choosing one curve over another or not allowing these trade-off curves to evolve as well. Another approach that has been much less explored would be to introduce a distribution of mutation effects, where specialist mutations are much more common than generalist mutations (having to solve the problem of a single habitat) and are constantly appearing at different loci maintaining local adaptation. In the latter situation, and if no other constraint is involved, the outcome in the very long run would be nearly perfect adaptation to the different habitats. There are however three positive feedback loops that are likely to interfere with this outcome and favor increased specialization.

2.3. The Demographic Feedback Loop. The first feedback is demographic. As far as local adaptation causes a local increase in density, it will also make life easier for more specialist alleles to increase in frequency. This is due to 
the fact that density differences cause asymmetric gene flow, which gives an advantage to alleles favored in the denser habitat $[14,22]$. Because of gene flow, an allele too detrimental outside the habitat where it is favored may be unable to increase in frequency. Despite having the potential to contribute to specialization if the habitat was isolated, it remains at mutation-selection balance. Such alleles may be very common; I term them "contending" alleles. If density becomes higher in this habitat, contending alleles may now be able to increase in frequency and contribute to local adaptation. This increase in density is likely to occur at least in some cases when local adaptation takes place. The positive feedback loop occurs because a local increase in density causes more and more alleles that are locally beneficial to be recruited, which strengthens local adaptation, increases local density and facilitates further the increase in frequency of other locally beneficial alleles (the reverse can also occur, which is known as migration meltdown [14, 39]). Because the ratio of density is as effective as the square of selection ratio inside versus outside the habitat $[14,22]$, this effect is likely to be strong in natural populations. Conversely adapting to a sink habitat makes it very difficult for the same reason as shown by niche expansion models [40-42].

2.4. The Recombination Feedback Loop. The second feedback loop is due to indirect selection among loci directly involved in local adaptation. When a locally beneficial allele increases in frequency, it will favor the spread at closely linked loci of other locally beneficial alleles. This is due to the fact that dispersal generates linkage disequilibrium between loci that share a similar frequency variation across habitats, as expected for two loci involved in local adaptation to the same habitat. This linkage disequilibrium translates into indirect selection that mutually benefits the locally adapted alleles at the two loci $[43,44]$. For instance, a contending allele could start to increase in frequency if it becomes sufficiently linked to another locus involved in the local adaptation. This phenomenon generates a positive feedback loop within the genome where locally adapted alleles are more likely to be recruited in genomic regions already harboring a previous locus involved in the local adaptation. It can generate "genomic islands" of local adaptation that extend further and further $[45,46]$, a specific process that can gradually produce strong genetic divergence at many contiguous loci in linkage disequilibrium $[1,47]$.

2.5. The Reinforcement Feedback Loop. The third feedback loop is due to reinforcement, that is, the evolution of traits promoting premating isolation between differentially locally adapted genotypes. Reinforcement tends to make life easier for locally beneficial alleles: it allows more alleles contributing to local adaptation to be recruited and locally beneficial alleles to reach higher frequencies. For instance, contending alleles could be recruited if habitat choice started to evolve. In effect, habitat choice minimizes the possible negative fitness that an allele can have in habitats that are different from the habitat where it is favored. Thus, reinforcement is likely to promote increased specialization.
Reciprocally, strong local adaptation increases the selection pressure to reinforce it, so that both phenomena can act in concert in a positive feedback loop $[9,37,38,48]$.

2.6. The Relative Dynamics of Local Adaptation and Reinforcement. Ultimately, ecological speciation will result only if reinforcement occurs quickly enough compared to the evolution of generalists and the breakdown of local adaptation. The different feedback loops mentioned above will tend to favor this outcome, but may not be strong enough to lead to speciation. For instance, the evolution of habitat choice, reduced dispersal, selfing, and so forth, can strongly reduce the chance that a generalist allele would spread, but the actual outcome depends on the relative rates of reinforcement and loss of specialization. This dynamical issue is not something that has been fully appreciated in the context of ecological speciation (but see [38] in the context of a fixed tradeoff). It differs from the situation of reinforcement in a tension zone by the fact that it is less stable and very sensitive to several feedbacks. More work is certainly needed to clearly delineate the conditions favoring speciation in this context.

\section{Topic 2-The Reinforcement of Local Adaptation}

"Individuals that have parents selected in the same habitat and that stay in that habitat are more likely to have genes appropriate to that environment than another randomly chosen individual. Thus, mating locally and staying in the same habitat is always favored from the point of view of a continually evolving genome" [25].

In the early 70s, several models have addressed the problem of reinforcement of local adaptation. The first was proposed by Antonovics [49]. This model showed that, because mating at random is risky in the context of local adaptation, evolution favors that like mates with like and that selfing is even safer to maintain local adaptation. Then Balkau and Feldman [50] showed that, in the context of local adaptation, migrating, or sending offspring elsewhere is likely to decrease an individual's fitness or that of its offspring. With a modifier model, they showed that this effect caused indirect selection to reduce dispersal as much as possible. Finally, Slatkin [43] suggested and D. Charlesworth and B. Charlesworth confirmed [51] that sex and recombination is likely to break combination of genes that have been locally selected for and should be selected against in the context of local adaptation. These findings have since been constantly reported or given as examples of "one-allele" mechanisms that are likely to drive the evolution of isolation in parapatry. (The one-allele versus two-allele classification refers to cases where a single or two different alleles spread at the modifier locus to promote genetic clustering [21]. This classification is very useful despite leading to some complications (see [52] and Table 1 note 7)). In this section, I will reconsider these conclusions in the light of more recent models on the evolution of assortative 
TABLE 1: Classification of reinforcement mechanisms based on the life stage modified and their consequences on genetic associations involved in genetic clustering. This classification is orthogonal to the one- versus two-allele classification.

\begin{tabular}{|c|c|c|c|}
\hline Life stage modified & Dispersal & Syngamy & Meiosis \\
\hline $\begin{array}{l}\text { Prominent biological } \\
\text { example }\end{array}$ & Habitat choice & Mate choice & Sex/asex \\
\hline $\begin{array}{l}\text { Genetic clustering at local } \\
\text { adaptation loci through }\end{array}$ & $\begin{array}{l}\text { Increased frequency } \\
\text { differences between } \\
\text { habitats }\end{array}$ & Increased homozygosity & Increased linkage disequilibrium \\
\hline $\begin{array}{l}\text { Usual population genetic } \\
\text { parameter measuring } \\
\text { clustering }\end{array}$ & $F_{\mathrm{st}}($ differentiation $)$ & $F_{\text {is }}($ departure from Hardy-Weinberg) & $D$ (linkage disequilibrium) \\
\hline $\begin{array}{l}\text { Primary effect of modifier } \\
\text { on local adaptation loci }\end{array}$ & Change in frequency & Change in within-locus associations ${ }^{2}$ & Change in between-loci associations \\
\hline $\begin{array}{l}\text { Primary modifier } \\
\text { association }^{3}\end{array}$ & $C_{\mathrm{ma}, \varnothing}$ & $C_{\mathrm{ma}, \mathrm{a}}$ & $C_{\mathrm{mab}, \varnothing}$ \\
\hline Increased differentiation & Directly & Indirectly ${ }^{4}$ & Indirectly ${ }^{4}$ \\
\hline $\begin{array}{l}\text { Notable complication } \\
\text { preventing clustering }^{5}\end{array}$ & Kin selection & Recessivity $^{6}$ & Negative epistasis \\
\hline “One-allele” examples & $\begin{array}{l}\text { An allele reducing dispersal } \\
\text { or causing preference to } \\
\text { natal habitat (Figure 2) }\end{array}$ & $\begin{array}{l}\text { An allele causes assortment based on } \\
\text { self-similarity (Figure 1) }\end{array}$ & $\begin{array}{l}\text { An allele causes a reduction in } \\
\text { recombination (Figure } 4 \text { ) }\end{array}$ \\
\hline “Two-allele" examples & $\begin{array}{l}\text { Allele } 1 \text { causes preference to } \\
\text { habitat } 1 \text { and allele } 2 \text { to } \\
\text { habitat } 2\end{array}$ & $\begin{array}{l}\text { Allele } 1 \text { cause preference to phenotype } \\
1 \text { and allele } 2 \text { to phenotype } 2^{7}\end{array}$ & $\begin{array}{l}\text { Allele } 1 \text { (inversion) causes linkage } \\
\text { in group of genes } 1 \text { and allele } 2 \\
\text { (noninversion) in group of genes } 2\end{array}$ \\
\hline
\end{tabular}

\footnotetext{
${ }^{1}$ This would also apply to loci involved in genetic incompatibilities in a secondary contact.

${ }^{2}$ Unless mating is selective and causes a direct advantage to locally adapted alleles (i.e., it changes frequency at the local adaptation loci), as found in model involving sexual selection $[8,103]$.

${ }^{3}$ Notation as in $[104,105]$, where $\mathrm{m}$ is the modifier locus and a, b the local adaptation loci. "Primary" association refers to the fact that the phenotypic effect of the modifier causes first a direct change on the genetic composition of the population (on frequency, within or between loci associations), which may then change the efficacy of selection. Eventually, a modifier promoting clustering will end up associated to the beneficial allele locally $\left(C_{\mathrm{ma}, \varnothing}>0\right)$. See Figures 1,2 and 4 for examples.

${ }^{4}$ Indirectly by increasing the variance in fitness and the efficacy of selection.

${ }^{5}$ Besides possible direct costs relative to the strategy used (e.g., cost of finding a mate or the right habitat). Different traits are exposed to a variety of other selective effects (see text).

${ }^{6}$ Which generates inbreeding depression.

${ }^{7}$ Phenotype 1 and 2 may result from alleles at the adaptation locus or to another unrelated marker trait. Similarly in a "one-allele" model, self-similarity may be evaluated in reference to a marker trait at another locus than the modifier or the local adaptation locus. In both cases, the marker trait has to diverge in the two incipient species, which is essentially a two-allele mechanism. Thus, with three locus like this, the one- versus two-allele distinction is made more complicated by the fact that the marker trait must diverge (two-allele), but the modifier of the strength of assortment need not (it can be one- or two-allele) [52]. Another complication of the one- and two-allele classification arises when the locus exposed to postzygotic selection also causes premating isolation (as seen in socalled "magic trait" models). This can be thought as the limit where the loci causing prezygotic isolation and postzygotic selection become confounded.
}

mating [53], dispersal $[54,55]$, and recombination $[11,45$, $56]$ in the context of local adaptation. Contrary to what is commonly thought $[8,21,57]$, I will show that these oneallele mechanisms do not inevitably lead to speciation, even in the absence of direct cost. I will then make a comparison of the underlying mechanisms and propose a typology of cases (orthogonal to the one- versus two-allele classification) that may prove useful understanding and modeling speciation (Table 1).

Before proceeding, we note that several important findings have also been made regarding this process since these early models. First, the role of ecological-based adaptation in speciation has received considerable support in the last decade $[2,4,9,10]$. Second several empirical findings have supported the idea that reinforcement could indeed occur in the context of adaptation to different habitats $[49,58-$ 67] or at least that there is often ample opportunity for reinforcement [6].
3.1. The Evolution of Selfing and Assortative Mating. The evolution of nonrandom mating has been extensively studied in the context of reinforcement and reproductive isolation $[18,68-70]$. However, it has also been extensively studied to understand the evolution of mating systems within species [71-73]. Interestingly, the two approaches are usually considered separately and emphasize completely opposite outcomes. The first predicts the evolution of more assortative mating with increased outbreeding depression or hybrid unfitness. The second predicts the evolution of less assortative mating or selfing with increased inbreeding depression. The models studying reinforcement include outbreeding but not inbreeding depression $[18,70,74-77]$ while the models studying mating system evolution do exactly the opposite [71-73].

Local adaptation causes outbreeding depression if different alleles are favored in different habitats [78], which is the reason why it is widely thought that spatially heterogenous 


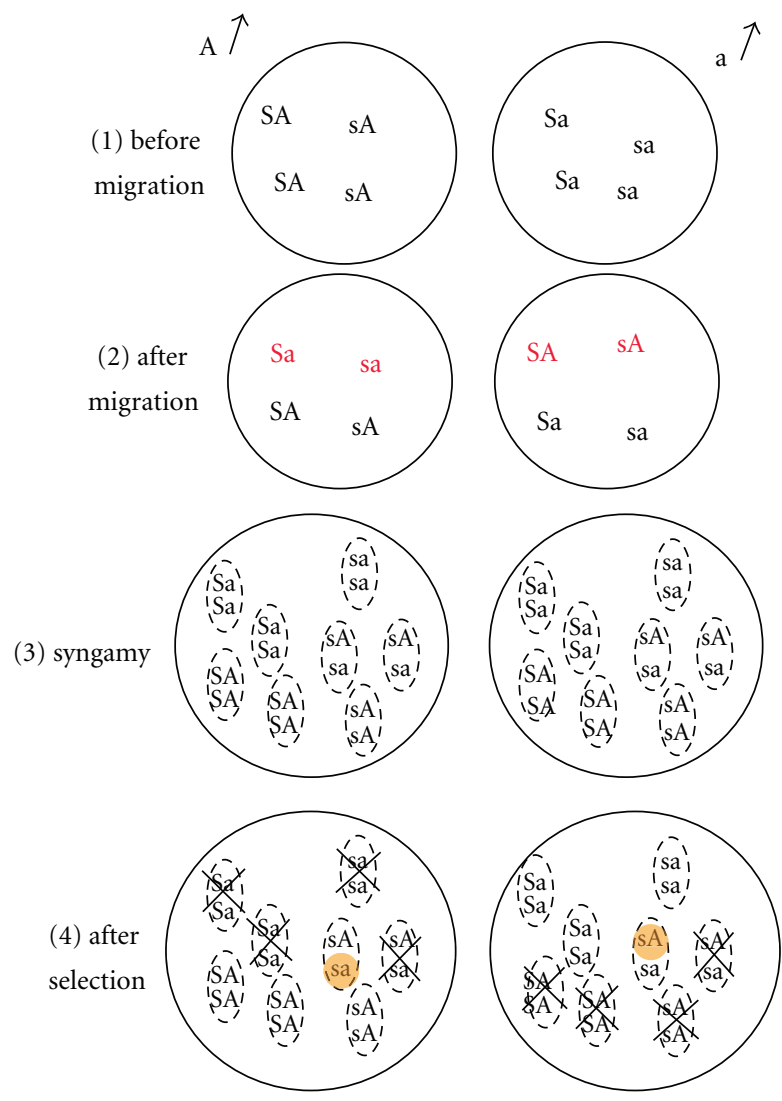

FIGURE 1: Indirect selection on a selfing/assortative mating modifier with local adaptation [53]. Sketches how a selfing or assortative mating modifier evolves in presence of local adaptation (for the sake of illustration, the $\mathrm{S}$ allele causes maximal assortment and s specifies random mating). Here assortment can be produced by selfing or assortative mating based on the genotype at the local adaptation locus (A mates with A and a with a). Before migration (step 1), consider two habitats with haploid individuals. On the left A allele is favored at a local adaptation locus, whereas a is favored on the right. To make things simple, we consider these alleles to be fixed where they are beneficial. At step 2, migration occurs between habitats (with $\mathrm{m}=1 / 2$, migrants in red). Then syngamy occurs in each habitat. The small circles represent diploid individuals. In each habitat, one can distinguish the subpopulation with full S-assortment allele (4 individuals on the left) and random mating s allele (4 individuals on the right). Importantly, at this step the S allele becomes positively associated to extreme aa and AA homozygotes (thus, variance in fitness is greater in the subpopulation with the $\mathrm{S}$ allele). Finally, selection occurs (favoring A on the left and a on the right, very strongly but in a codominant way in the illustration). At the end of this generation, the modifier has not changed in frequency (it is still 1/2). Yet, selection has generated LD between the random mating s allele and the locally inferior allele locally (the inferior allele is only found on the same chromosome as s in each habitat, orange dot). At the next generation, this LD will persist (it is decreased at most by one half by a round of free recombination) and cause indirect selection in favor of S. Note that if the locally beneficial allele is recessive (all heterozygotes eliminated on the right and the left), we see that direct selection occurs favoring S (its frequency rises to $2 / 3$ on the sketch), but that less LD is generated. Exactly the opposite occurs if the local beneficial allele is dominant.

selection favors the evolution of assortative mating by a oneallele mechanism in the absence of direct costs $[8,18,21,68$, $69,79,80]$. However, the dominance relationship of locally adapted alleles within each habitat may also cause inbreeding depression which can, in fact, prevent the evolution of premating isolation. When both phenomena act in concert, the outcomes vary tremendously depending on parameter values [53], and more assortment is not necessarily favored even in presence of strong local adaptation. In fact, because a polymorphism at a locus involved in local adaptation is more easily maintained when locally beneficial alleles are dominant, less assortment may often be favored in natural situations with local adaptation [53]. There are theoretical reasons for expecting such dominance relationships between locally adapted alleles [81]. Because theoretical models of reinforcement have rarely considered the case of local adaptation, and when they did, considered only haploid or diploid with particular dominance $[18,70,82]$, these conclusions have remained largely overlooked. There are numerous mechanisms of assortment [69] and each can evolve slightly differently. For instance when local adaptation is based on a conspicuous trait (shell thickness in Littorina [65], coloration in Chrysopa [62] or Heliconius [83], etc.), mate choice can be cued directly on this trait, which is very efficient unless the right mate is rare and difficult to find in the population [84]. Another simple way to mate with a selfsimilar phenotype is to self-fertilize when hermaphrodite which is also very efficient, does not incur the cost of finding the right mate and does not require the ability to discriminate the locally adapted trait. In both cases, local adaptation 


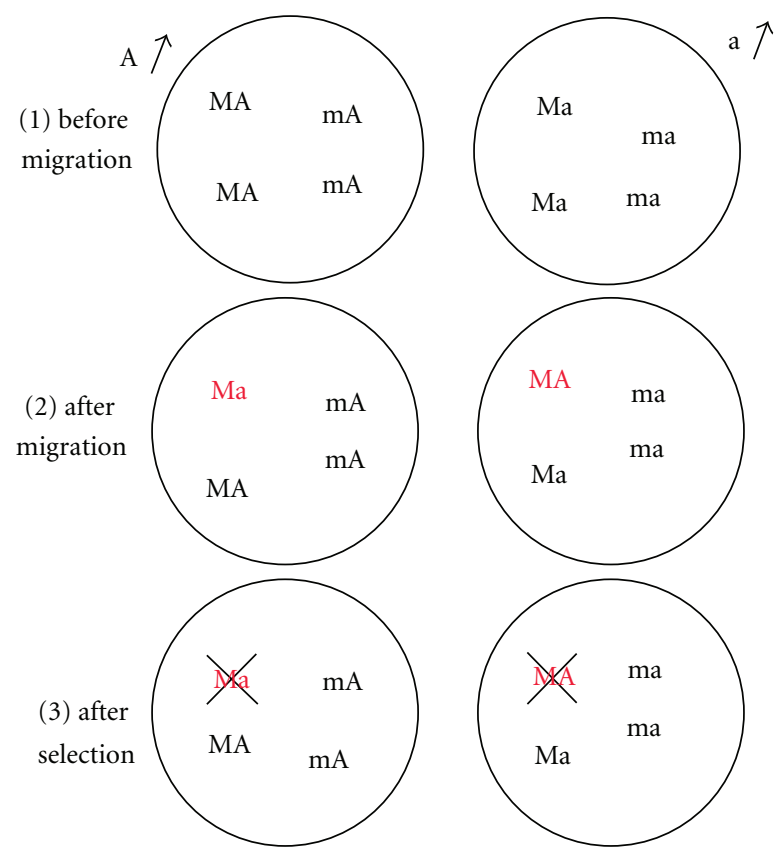

FIGURE 2: Indirect selection on a migration modifier with local adaptation. Sketches how a migration modifier evolves in presence of local adaptation (for the sake of illustration the $\mathrm{M}$ allele causes maximal migration (1/2) and the $\mathrm{m}$ allele zero migration). Before migration (step 1) consider two habitats with haploid individuals. Before migration (step 1) consider two habitats with haploid individuals. On the left the A allele is favored at a local adaptation locus whereas a is favored on the right. To make things simple we consider these alleles to be fixed where they are beneficial. During migration only individuals with allele M move between habitats (step (2) migrants in red). Half of the M individuals move to the other habitat and the other half stays at home. Importantly migration directly generates LD between the M allele and the locally inferior allele (the locally inferior allele is found only with $\mathrm{M}$ and not with $\mathrm{m}$ ). Finally, selection occurs favoring A on the left and a on the right very strongly in the illustration and carries the $\mathrm{m}$ allele with the adaptation locus because of the linkage disequilibrium generated at the previous step (the m overall frequency has raised from $1 / 2$ to $2 / 3$ on the illustration). Note that in a finite population kin selection by contrast favors $\mathrm{M}$ [54].

loci will cause indirect selection (Figure 1) and, if not codominant, inbreeding depression. With selfing however, all other loci in the genome experiencing recessive deleterious mutations will also contribute to inbreeding depression. As a consequence, and unless the cost of finding a mate is high, reinforcement may be less likely to evolve via selfing than via assortment based on the local adaptation traits [53]. Twoallele models and models involving sex-specific traits and preferences also provide several alternatives $[8,52]$.

3.2. The Evolution of Dispersal. Individuals that have survived until reproduction have genotypes that work relatively well where they are. Because of environmental heterogeneity, migrating or sending offspring elsewhere is likely to decrease fitness. Local adaptation indeed generates an indirect selection pressure in favor of less dispersal $[50,85-$ 87] (Figure 2). However, as for the evolution of nonrandom mating, the evolution of dispersal has been studied in a variety of contexts and not only in reference to the process of the reinforcement of local adaptation or speciation and a large number of factors interact to shape this trait [88]. However, in the context of the evolution of dispersal in presence of local adaptation there are at least two factors that cannot be ignored. The first is that, as in the case of the evolution of assortment, inbreeding depression causes a selection pressure in favor of dispersal [89]. This inbreeding depression can be partly, but not only, caused by the loci responsible for the local adaptation. The second factor is kin selection (Figure 3). As soon as one considers a stochastic model for the evolution of dispersal, kin selection occurs and must be taken into account to determine how dispersal evolves [90-92]. Intuitively, it is straightforward to see that a given allele causing zero dispersal cannot fix in a subdivided population. In other words, zero dispersal cannot be a convergent stable state as was suggested in deterministic models of reinforcement. As expected from this heuristic argument, kin selection favors more dispersal than predicted in a deterministic model [54]. However, this is not the only effect as local adaptation interacts with the effect of kin selection: strong differentiation at a local adaptation locus magnifies kin selection at short recombination distance. This indirect kin selection can cause bistability (i.e., different dispersal rate can evolve depending on the initial conditions), which changes qualitatively the expectation [54]. There are different ways to reduce dispersal, and all may not be equivalent even if the selection pressures at work will share strong similarities. In particular it is clearly important to distinguish between dispersal and habitat choice. As we have seen dispersal cannot evolve to very low rates because 


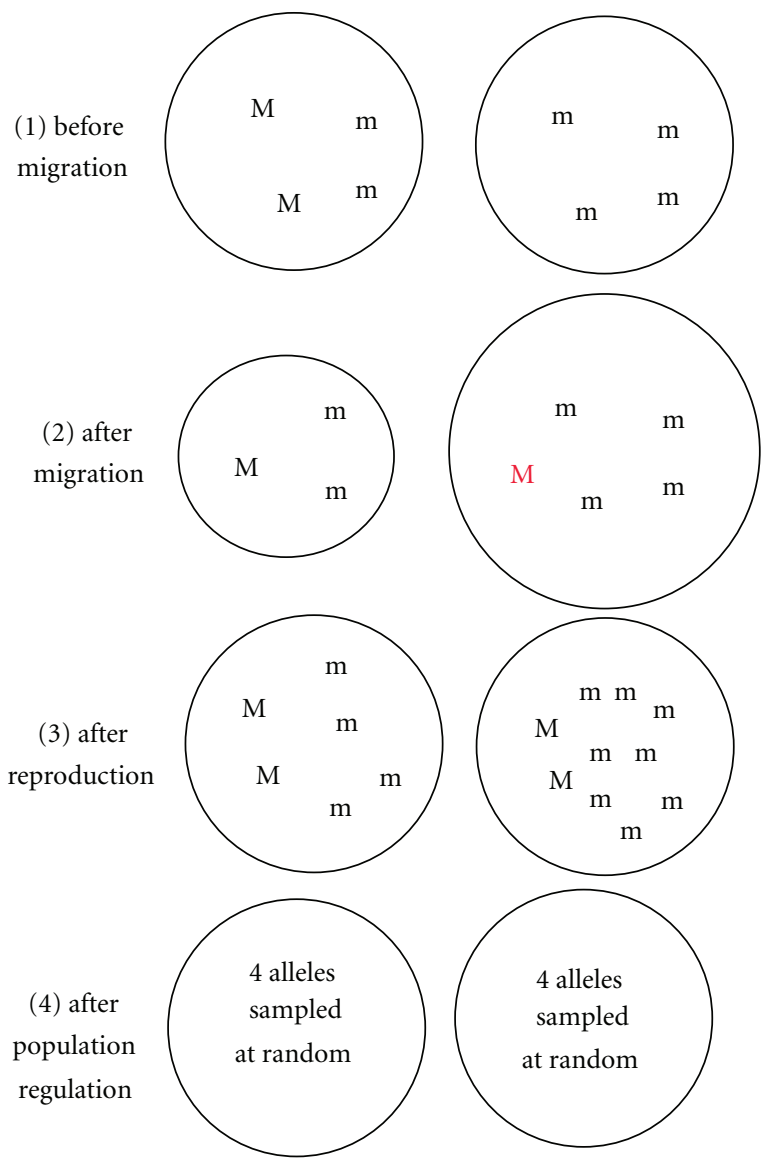

FIGURE 3: Kin selection on a migration modifier. Sketches how a migration modifier evolves because of kin selection. As in Figure 2, the M allele causes maximal migration (1/2) and the $\mathrm{m}$ allele specifies zero migration). Before migration (step 1), consider two subpopulations. In one of them the M allele is frequent (1/2), but it is absent in the other. During migration, only M individuals move between habitats (step (2), the migrant is shown in red). Half of the M individuals move to the other habitat and the other half stays at home. Then reproduction occurs (step 3). All individuals produce say, two offsprings (note that all individuals have the same survival and reproduction). Finally, population regulation occurs: juveniles compete to repopulate each subpopulation with four adults and all have the same chance to get established. After this step, the $\mathrm{M}$ frequency has risen to $(1 / 3+1 / 5) / 2$, which is greater than $1 / 4$, the initial frequency. There is thus selection on $M$ allele, which is traditionally explained in terms of "kin selection": the migrating M allele sacrifices itself by competing in a more crowded population, but it leaves room behind that benefits the other $\mathrm{M}$ allele, which will compete in a less crowded population. The decreased chance of survival by the migrating $\mathrm{M}(1 / 5-1 / 4)$ is more than compensated by the increased chance that the remaining $\mathrm{M}$ allele will survive (1/3-1/4). This process requires only that the $\mathrm{M}$ alleles are concentrated in the same population at step 1 (i.e., it requires population structure or relatedness), which is easily generated by drift [54].

of kin selection. However, choosing the natal habitat (to maintain local adaptation) while quitting its natal patch (to release kins from competition) may provide the best from both worlds and is therefore a more likely candidate trait for reinforcement. Two-allele models also provide several alternatives $[38,57]$.

3.3. Comparisons among Reinforcement Traits. The common effect in all these processes is that alleles that favor more assortment, less dispersal or tighter linkage become associated with locally beneficial alleles, which in turn generates an indirect selection pressure in their favor. In each of these cases however, the way linkage disequilibrium is built between the modifiers and the locally beneficial alleles is distinct (compare Figures 1, 2 and 4). First, a modifier has an immediate effect on the genetic composition of the population, here genotypic frequencies at the local adaptation loci: dispersal modification changes allelic frequencies; assortment changes within locus associations; recombination changes between loci associations. This immediate effect causes a frequency change at the modifier locus if there is selection on alleles, dominance, and epistasis, respectively. When the modifier changes within or between loci associations, a secondary effect occurs. Increased associations generate a higher variance in fitness, more efficient selection, and thus an increase of the frequency difference between habitats at the local adaptation locus. As a consequence, modifiers increasing these associations (modifier increasing assortment or reducing recombination in our examples) become associated, and hitchhike, with locally beneficial alleles. There 


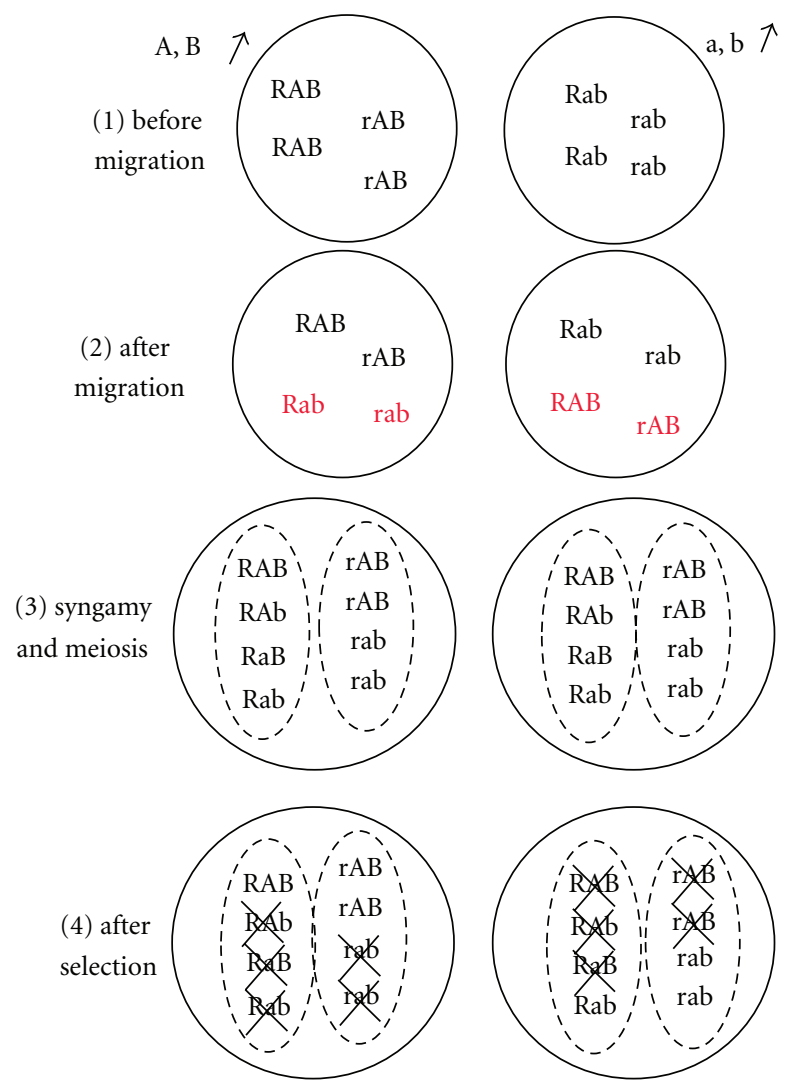

FIGURE 4: Indirect selection on a recombination modifier with local adaptation. Sketches how a recombination modifier evolves in presence of local adaptation (for the sake of illustration, the $\mathrm{R}$ allele causes maximal recombination, and the $\mathrm{r}$ allele specifies zero recombination). Before migration (step 1), consider two habitats with haploid individuals. On the left, the A and B alleles are favored at two different loci; the $\mathrm{a}$ and $\mathrm{b}$ alleles are favored on the right. To simplify the illustration, we consider the alleles to be fixed where they are beneficial. After migration between habitats ( $\operatorname{step}(2)$ with $\mathrm{m}=1 / 2$, migrants shown in red), strong LD is generated between the selected loci: in each habitat there are $\mathrm{AB}$ and $\mathrm{ab}$ but no $\mathrm{Ab}$ and $\mathrm{aB}$ haplotypes. Then random mating and meiosis occur (step 3 ). In each habitat, one can distinguish the subpopulation with the full $\mathrm{R}$ recombination allele and the zero $\mathrm{r}$ recombination allele (groups of four individuals on the left and right, resp.). Within the former, $\mathrm{LD}$ between selected loci has been much reduced (illustrated at zero, in fact even full recombination only halves $\mathrm{LD}$ at each meiosis), whereas in the latter it stayed intact. Importantly, at this step the r recombination modifier becomes positively associated to the extreme $\mathrm{AB}$ and ab haplotypes. Note also that in each habitat, variance in fitness is greater in the subpopulation with the $\mathrm{r}$ allele. Finally, selection occurs (favoring $\mathrm{A}$ and $\mathrm{B}$ on the left and $\mathrm{a}$ and $\mathrm{b}$ on the right, very strongly on the illustration) and takes the $\mathrm{r}$ allele along because of the linkage disequilibrium generated at the previous step (the overall frequency of $r$ has risen from $1 / 2$ to $2 / 3$ in the illustration). Note that the case illustrated involves positive epistasis (only the extreme genotypes $\mathrm{AB}$ and ab survive, on the left and right, resp.). However, the $r$ allele is favoured even if epistasis is zero, because selection is more efficient in subpopulations carrying the $r$ allele since variance in fitness is greater in these subpopulations.

are thus several ways to promote distinct genetic clusters between habitats and the three examples detailed in this paper illustrate each of these cases: directly magnifying allelic frequency differences between populations (case illustrated by dispersal modification), promoting within locus associations (case illustrated by assortment modification), promoting between loci associations (case illustrated by recombination modification). Reinforcement may occur by the evolution of many other traits than the ones mentioned here (in particular involving two-allele mechanisms, see Table 1), but their impact are likely to be achieved via one of these effects alone or in combination. Considering the three possible impacts of a modifier on the genetic composition of populations (on frequencies, within locus and between loci associations) may be a useful typology to understand the different ways reinforcement and genetic clustering can occur. It is orthogonal to, and complements the usual oneversus two-allele classification (Table 1).

\section{Conclusion}

The first conclusion is that the process of reinforcement and local adaptation are intertwined and occur simultaneously. Whether pre- and postzygotic isolation will eventually evolve is uncertain in such a dynamic process. In particular, local adaptation can collapse if generalist alleles arise and spread. However, there are several positive feedback loops that will tend to drive the system towards divergence (the reinforcement, demographic, and recombination loops). 
From a theoretical standpoint, this process has rarely been analyzed jointly and in a dynamic way with changes in local adaptation itself. In the context of mounting evidence in favor of ecological speciation [106, 107], such an approach would certainly help evaluate its likelihood, tempo, and mechanism.

Second, Felsenstein [21] proposed to distinguish the different mechanisms for reinforcement on the idea that they involved the spread of one or two-alleles in the incipient species. This distinction is an important one, but it is not the only one to be made. Many one-allele mechanisms are only superficially similar as they can promote genetic clustering and speciation in different ways. A useful typology could be that they increase differentiation among populations, heterozygote deficit, or linkage disequilibrium, which corresponds to modifying one of the three fundamental events in a sexual life cycle (dispersal, syngamy, or meiosis, resp.). Furthermore, different traits may increase genetic clustering, but may not contribute to reinforcement because they are exposed to a variety of other selective effects. Models of reinforcement based on the evolution of particular traits must integrate what is known outside the speciation literature for those traits. For instance, recombination [108], mating systems [109], and dispersal [110], as discussed above, have all been intensely studied outside this context pinpointing a variety of selective effects. These theoretical developments certainly have to be merged.

\section{Acknowledgments}

I thank A. Whibley, J. Mallet and two anonymous reviewers for comments on this manuscript. This work was supported by the European Research Council starting grant "QuantEvol".

\section{References}

[1] C. I. Wu, "The genic view of the process of speciation," Journal of Evolutionary Biology, vol. 14, no. 6, pp. 851-865, 2001.

[2] M. Schilthuizen, "Dualism and conflicts in understanding speciation," BioEssays, vol. 22, no. 12, pp. 1134-1141, 2000.

[3] S. Gavrilets, "Perspective: models of speciation: what have we learned in 40 years?" Evolution, vol. 57, no. 10, pp. 2197 2215, 2003.

[4] C. D. Jiggins and J. Mallet, "Bimodal hybrid zones and speciation," Trends in Ecology and Evolution, vol. 15, no. 6, pp. 250-255, 2000.

[5] J. Mallet, "A species definition for the modern synthesis," Trends in Ecology and Evolution, vol. 10, no. 7, pp. 294-299, 1995.

[6] J. Mallet, "Hybridization as an invasion of the genome," Trends in Ecology and Evolution, vol. 20, no. 5, pp. 229-237, 2005.

[7] N. H. Barton and B. Charlesworth, "Genetic revolutions, founder effects, and speciation," Annual Review of Ecology and Systematics, vol. 15, pp. 133-164, 1984.

[8] M. Kirkpatrick and V. Ravigné, "Speciation by natural and sexual selection: models and experiments," The American Naturalist, vol. 159, supplement 3, pp. S22-S35, 2002.
[9] S. Via, "Sympatric speciation in animals: the ugly duckling grows up," Trends in Ecology and Evolution, vol. 16, no. 7, pp. 381-390, 2001.

[10] D. Schluter, "Ecology and the origin of species," Trends in Ecology and Evolution, vol. 16, no. 7, pp. 372-380, 2001.

[11] M. Kirkpatrick and N. Barton, "Chromosome inversions, local adaptation and speciation," Genetics, vol. 173, no. 1, pp. 419-434, 2006.

[12] M. Arnold, Natural Hybridization and Evolution, Oxford University Press, Oxford, UK, 1997.

[13] L. E. B. Kruuk, S. J. E. Baird, K. S. Gale, and N. H. Barton, "A comparison of multilocus clines maintained by environmental adaptation or by selection against hybrids," Genetics, vol. 153, no. 4, pp. 1959-1971, 1999.

[14] T. Lenormand, "Gene flow and the limits to natural selection," Trends in Ecology and Evolution, vol. 17, no. 4, pp. 183189, 2002.

[15] T. Nagylaki, "Conditions for the existence of clines," Genetics, vol. 80, no. 3, pp. 595-615, 1975.

[16] M. Slatkin, "Gene flow and selection in a cline," Genetics, vol. 75, no. 4, pp. 733-756, 1973.

[17] N. H. Barton and G. M. Hewitt, "Analysis of hybrid zones," Annual Review of Ecology and Systematics, vol. 16, pp. 113148, 1985.

[18] M. R. Servedio, "The evolution of premating isolation: local adaptation and natural and sexual selection against hybrids," Evolution, vol. 58, no. 5, pp. 913-924, 2004.

[19] J. Mallet, A. Meyer, P. Nosil, and J. L. Feder, "Space, sympatry and speciation," Journal of Evolutionary Biology, vol. 22, no. 11, pp. 2332-2341, 2009.

[20] F. Débarre and T. Lenormand, "Distance-limited dispersal promotes coexistence at habitat boundaries: reconsidering the competitive exclusion principle," Ecology Letters, vol. 14, no. 3, pp. 260-266, 2011.

[21] J. Felsenstein, "Skepticism towards Santa Rosalia, or why are there so few kinds of animals?" Evolution, vol. 35, no. 1, pp. 124-138, 1981 .

[22] T. Nagylaki, "Clines with asymmetric migration," Genetics, vol. 88, no. 4, pp. 813-827, 1978.

[23] P. Labbé, N. Sidos, M. Raymond, and T. Lenormand, "Resistance gene replacement in the mosquito Culex pipiens: fitness estimation from long-term cline series," Genetics, vol. 182, no. 1, pp. 303-312, 2009.

[24] T. J. Kawecki, N. H. Barton, and J. D. Fry, "Mutational collapse of fitness in marginal habitats and the evolution of ecological specialisation," Journal of Evolutionary Biology, vol. 10, no. 3, pp. 407-429, 1997.

[25] M. C. Whitlock, “The red queen beats the jack-of-all-trades: the limitations on the evolution of phenotypic plasticity and niche breadth," The American Naturalist, vol. 148, supplement, pp. S65-S77, 1996.

[26] M. C. Hall, D. B. Lowry, and J. H. Willis, "Is local adaptation in Mimulus guttatus caused by trade-offs at individual loci?" Molecular Ecology, vol. 19, no. 13, pp. 2739-2753, 2010.

[27] F. M. Cohan, E. C. King, and P. Zawadzki, "Amelioration of the deleterious pleiotropic effects of an adaptive mutation in Bacillus subtilis," Evolution, vol. 48, no. 1, pp. 81-95, 1994.

[28] T. Guillemaud, T. Lenormand, D. Bourguet, C. Chevillon, N. Pasteur, and M. Raymond, "Evolution of resistance in Culexpipiens: allele replacement and changing environment," Evolution, vol. 52, no. 2, pp. 443-453, 1998.

[29] J. B. S. Haldane, The Causes of Evolution, Harper, New York, NY, USA, 1932. 
[30] P. Labbe, T. Lenormand, and M. Raymond, "On the worldwide spread of an insecticide resistance gene: a role for local selection," Journal of Evolutionary Biology, vol. 18, no. 6, pp. 1471-1484, 2005.

[31] R. A. Fisher, "The possible modification of the response of the wild type to recurrent mutations," The American Naturalist, vol. 62, no. 679, pp. 115-126, 1928.

[32] R. E. Lenski, "Experimental studies of pleiotropy and epistasis in Escherichia coli-II. Compensation for maladaptive effects associated with resistance to virus T4," Evolution, vol. 42, no. 3, pp. 433-440, 1988.

[33] J. A. McKenzie, Ecological and Evolutionary Aspects of Insecticide Resistance, R. G. Landes, Austin, Tex, USA, 1996.

[34] P. Labbé, A. Berthomieu, C. Berticat et al., "Independent duplications of the acetylcholinesterase gene conferring insecticide resistance in the mosquito Culex pipiens," Molecular Biology and Evolution, vol. 24, no. 4, pp. 1056-1067, 2007.

[35] P. Labbé, C. Berticat, A. Berthomieu et al., "Forty years of erratic insecticide resistance evolution in the mosquito Culex pipiens," PLoS Genetics, vol. 3, no. 11, Article ID e205, pp. 2190-2199, 2007.

[36] T. Lenormand, T. Guillemaud, D. Bourguet, and M. Raymond, "Appearance and sweep of a gene duplication: adaptive response and potential for new functions in the mosquito Culex pipiens," Evolution, vol. 52, no. 6, pp. 1705-1712, 1998.

[37] M. Egas, U. Dieckmann, and M. W. Sabelis, "Evolution restricts the coexistence of specialists and generalists: the role of trade-off structure," The American Naturalist, vol. 163, no. 4, pp. 518-531, 2004.

[38] V. Ravigné, U. Dieckmann, and I. Olivieri, "Live where you thrive: joint evolution of habitat choice and local adaptation facilitates specialization and promotes diversity," The American Naturalist, vol. 174, no. 4, pp. E141-E169, 2009.

[39] O. Ronce and M. Kirkpatrick, "When sources become sinks: migrational meltdown in heterogeneous habitats," Evolution, vol. 55 , no. 8 , pp. $1520-1531,2001$.

[40] R. Gomulkiewicz, R. D. Holt, and M. Barfield, "The effects of density dependence and immigration on local adaptation and niche evolution in a black-hole sink environment," Theoretical Population Biology, vol. 55, no. 3, pp. 283-296, 1999.

[41] N. Barton, "Adaptation at the edge of a species' range," in Integrating Genetics and Ecology in a Spatial Context, J. Antonovics and J. Silvertown, Eds., pp. 365-392, Blackwell, London, UK, 2001.

[42] M. Kirkpatrick and N. H. Barton, "Evolution of a species' range," The American Naturalist, vol. 150, no. 1, pp. 1-23, 1997.

[43] M. Slatkin, "Gene flow and selection in a two locus system," Genetics, vol. 81, no. 4, pp. 787-802, 1975.

[44] T. Lenormand, T. Guillemaud, D. Bourguet, and M. Raymond, "Evaluating gene flow using selected markers: a case study," Genetics, vol. 149, no. 3, pp. 1383-1392, 1998.

[45] T. Lenormand and S. P. Otto, "The evolution of recombination in a heterogeneous environment," Genetics, vol. 156, no. 1, pp. 423-438, 2000.

[46] S. Yeaman and M. C. Whitlock, "The genetic architecture of adaptation under migration-selection balance," Evolution, vol. 65, no. 7, pp. 1897-1911, 2011.

[47] C. I. Wu and C. T. Ting, "Genes and speciation," Nature Reviews Genetics, vol. 5, no. 2, pp. 114-122, 2004.

[48] U. Dieckmann and M. Doebeli, "On the origin of species by sympatric speciation,” Nature, vol. 400, no. 6742, pp. 354357, 1999.
[49] J. Antonovics, "Evolution in closely adjacent plant populations-V. Evolution of self-fertility," Heredity, vol. 23, no. 2, pp. 219-238, 1968.

[50] B. J. Balkau and M. W. Feldman, "Selection for migration modification,” Genetics, vol. 74, no. 1, pp. 171-174, 1973.

[51] D. Charlesworth and B. Charlesworth, "Selection of recombination in clines," Genetics, vol. 91, no. 3, pp. 581-589, 1979.

[52] M. R. Servedio, "The role of linkage disequilibrium in the evolution of premating isolation," Heredity, vol. 102, no. 1, pp. 51-56, 2009.

[53] G. Epinat and T. Lenormand, "The evolution of assortative mating and selfing with in- and outbreeding depression," Evolution, vol. 63, no. 8, pp. 2047-2060, 2009.

[54] S. Billiard and T. Lenormand, "Evolution of migration under kin selection and local adaptation," Evolution, vol. 59, no. 1, pp. 13-23, 2005.

[55] P. R. Armsworth, "Conditional dispersal, clines, and the evolution of dispersiveness," Theoretical Ecology, vol. 2, no. 2, pp. 105-117, 2009.

[56] K. V. Pylkov, L. A. Zhivotovsky, and M. W. Feldman, "Migration versus mutation in the evolution of recombination under multilocus selection," Genetical Research, vol. 71, no. 3, pp. 247-256, 1998.

[57] J. D. Fry, "Multilocus models of sympatric speciation: bush versus rice versus felsenstein," Evolution, vol. 57, no. 8, pp. 1735-1746, 2003.

[58] R. Hopkins and M. D. Rausher, "Identification of two genes causing reinforcement in the Texas wildflower Phlox drummondii," Nature, vol. 469, pp. 411-414, 2011.

[59] J. T. Anderson, J. H. Willis, and T. Mitchell-Olds, "Evolutionary genetics of plant adaptation," Trends in Genetics, vol. 27, no. 7, pp. 258-266, 2011.

[60] J. Hollander, M. Lindegarth, and K. Johannesson, "Local adaptation but not geographical separation promotes assortative mating in a snail," Animal Behaviour, vol. 70, no. 5, pp. 1209-1219, 2005.

[61] T. McNeilly and J. Antonovics, "Evolution in closely adjacent plant populations-IV. Barriers to gene flow," Heredity, vol. 23, no. 2, pp. 205-218, 1968.

[62] C. A. Tauber and M. J. Tauber, "Sympatric speciation based on allelic changes at three loci: evidence from natural populations in two habitats," Science, vol. 197, no. 4310, pp. 1298-1299, 1977.

[63] M. Macnair and P. Christie, "Reproductive isolation as a pleiotropic effect of copper tolerance in Mimulus guttatus?" Heredity, vol. 50, no. 3, pp. 295-302, 1983.

[64] S. Dubois, P. O. Cheptou, C. Petit et al., "Genetic structure and mating systems of metallicolous and nonmetallicolous populations of Thlaspi caerulescens," New Phytologist, vol. 157, no. 3, pp. 633-641, 2003.

[65] K. Johannesson, "Evolution in Littorina: ecology matters," Journal of Sea Research, vol. 49, no. 2, pp. 107-117, 2003.

[66] J. W. Grahame, C. S. Wilding, and R. K. Butlin, "Adaptation to a steep environmental gradient and an associated barrier to gene exchange in Littorina saxatilis," Evolution, vol. 60, no. 2, pp. 268-278, 2006.

[67] J. Antonovics, "Evolution in closely adjacent plant populations-X: long-term persistence of prereproductive isolation at a mine boundary," Heredity, vol. 97, no. 1, pp. 33-37, 2006.

[68] J. Coyne, H. Orr et al., Speciation, Sinauer Associates, Sunderland, Mass, USA, 2004.

[69] S. Gavrilets, Fitness Landscape and the Origin of Species, Princeton University Press, Princeton, NJ, USA, 2004. 
[70] M. R. Servedio, "Reinforcement and the genetics of nonrandom mating," Evolution, vol. 54, no. 1, pp. 21-29, 2000.

[71] R. Lande and D. W. Schemske, "The evolution of selffertilization and inbreeding depression in plants-I. Genetic models," Evolution, vol. 39, no. 1, pp. 24-40, 1985.

[72] D. Charlesworth, M. T. Morgan, and B. Charlesworth, "Inbreeding depression, genetic load, and the evolution of outcrossing rates in a multilocus system with no linkage," Evolution, vol. 44, no. 6, pp. 1469-1489, 1990.

[73] M. K. Uyenoyama and D. M. Waller, "Coevolution of self-fertilization and inbreeding depression-I. Mutationselection balance at one and two loci," Theoretical Population Biology, vol. 40, no. 1, pp. 14-46, 1991.

[74] M. Kirkpatrick, "Reinforcement and divergence under assortative mating," Proceedings of the Royal Society B, vol. 267, no. 1453, pp. 1649-1655, 2000.

[75] M. Kirkpatrick and M. R. Servedio, "The reinforcement of mating preferences on an island," Genetics, vol. 151, no. 2, pp. 865-884, 1999.

[76] S. P. Otto, M. R. Servedio, and S. L. Nuismer, "Frequencydependent selection and the evolution of assortative mating," Genetics, vol. 179, no. 4, pp. 2091-2112, 2008.

[77] M. R. Servedio and M. Kirkpatrick, "The effects of gene flow on reinforcement," Evolution, vol. 51, no. 6, pp. 1764-1772, 1997.

[78] H. D. Rundle and M. C. Whitlock, "A genetic interpretation of ecologically dependent isolation," Evolution, vol. 55, no. 1, pp. 198-201, 2001.

[79] J. A. Endler, Geographic Variation, Speciation and Clines, Princeton University Press, Princeton, NJ, USA, 1977.

[80] J. Maynard Smith, "Sympatric speciation," The American Naturalist, vol. 100, no. 916, pp. 637-650, 1966.

[81] S. P. Otto and D. Bourguet, "Balanced polymorphisms and the evolution of dominance," The American Naturalist, vol. 153, no. 6, pp. 561-574, 1999.

[82] S. Gavrilets, "The Maynard Smith model of sympatric speciation," Journal of Theoretical Biology, vol. 239, no. 2, pp. 172-182, 2006.

[83] C. D. Jiggins, R. E. Naisbit, R. L. Coe, and J. Mallet, "Reproductive isolation caused by colour pattern mimicry," Nature, vol. 411, no. 6835, pp. 302-305, 2001.

[84] M. Kirkpatrick and S. L. Nuismer, "Sexual selection can constrain sympatric speciation," Proceedings of the Royal Society B, vol. 271, no. 1540, pp. 687-693, 2004.

[85] S. Karlin and R. B. Campbell, "The existence of a protected polymorphism under conditions of soft as opposed to hard selection in a multideme population system," The American Naturalist, vol. 117, no. 3, pp. 262-275, 1981.

[86] P. Wiener and M. W. Feldman, "The effects of the mating system on the evolution of migration in a spatially heterogeneous population," Evolutionary Ecology, vol. 7, no. 3, pp. 251-269, 1993.

[87] J. H. Gillespie, "The role of migration in the genetic structure of populations in temporally and spatially varying environments-III. Migration modification," The American Naturalist, vol. 117, no. 3, pp. 223-233, 1981.

[88] O. Ronce, "How does it feel to be like a rolling stone? Ten questions about dispersal evolution," Annual Review of Ecology, Evolution, and Systematics, vol. 38, pp. 231-253, 2007.

[89] D. Roze and F. Rousset, "Inbreeding depression and the evolution of dispersal rates: a multilocus model," The American Naturalist, vol. 166, no. 6, pp. 708-721, 2005.
[90] W. D. Hamiltion and R. M. May, "Dispersal in stable habitats," Nature, vol. 269, no. 5629, pp. 578-581, 1977.

[91] S. A. Frank, "Dispersal polymorphisms in subdivided populations," Journal of Theoretical Biology, vol. 122, no. 3, pp. 303-309, 1986.

[92] P. D. Taylor, "An inclusive fitness model for dispersal of offspring," Journal of Theoretical Biology, vol. 130, no. 3, pp. 363-378, 1988.

[93] N. Barton and B. O. Bengtsson, "The barrier to genetic exchange between hybridising populations," Heredity, vol. 57, no. 3, pp. 357-376, 1986.

[94] N. H. Barton and B. Charlesworth, "Why sex and recombination?” Science, vol. 281, no. 5385, pp. 1986-1990, 1998.

[95] S. P. Otto and T. Lenormand, "Resolving the paradox of sex and recombination," Nature Reviews Genetics, vol. 3, no. 4, pp. 252-261, 2002.

[96] A. I. Khan, D. M. Dinh, D. Schneider, R. E. Lenski, and T. F. Cooper, "Negative epistasis between beneficial mutations in an evolving bacterial population," Science, vol. 332, no. 6034, pp. 1193-1196, 2011.

[97] H. H. Chou, H. C. Chiu, N. F. Delaney, D. Segrè, and C. J. Marx, "Diminishing returns epistasis among beneficial mutations decelerates adaptation," Science, vol. 332, no. 6034, pp. 1190-1192, 2011.

[98] D. R. Rokyta, P. Joyce, S. B. Caudle, C. Miller, C. J. Beisel, and H. A. Wichman, "Epistasis between beneficial mutations and the phenotype-to-fitness map for a ssDNA virus," PLoS Genetics, vol. 7, no. 6, Article ID e1002075, 2011.

[99] G. Martin, S. F. Elena, and T. Lenormand, "Distributions of epistasis in microbes fit predictions from a fitness landscape model," Nature Genetics, vol. 39, no. 4, pp. 555-560, 2007.

[100] N. H. Barton and S. P. Otto, "Evolution of recombination due to random drift," Genetics, vol. 169, no. 4, pp. 2353-2370, 2005.

[101] G. Martin, S. P. Otto, and T. Lenormand, "Selection for recombination in structured populations," Genetics, vol. 172, no. 1, pp. 593-609, 2006.

[102] D. Roze and N. H. Barton, "The Hill-Robertson effect and the evolution of recombination," Genetics, vol. 173, no. 3, pp. 1793-1811, 2006.

[103] M. A. R. de Cara, N. H. Barton, and M. Kirkpatrick, "A model for the evolution of assortative mating," The American Naturalist, vol. 171, no. 5, pp. 580-596, 2008.

[104] N. H. Barton and M. Turelli, "Natural and sexual selection on many loci," Genetics, vol. 127, no. 1, pp. 229-255, 1991.

[105] M. Kirkpatrick, T. Johnson, and N. Barton, "General models of multilocus evolution," Genetics, vol. 161, no. 4, pp. 17271750, 2002.

[106] D. Schluter, "Evidence for ecological speciation and its alternative," Science, vol. 323, no. 5915, pp. 737-741, 2009.

[107] J. Mallet, "Hybridization, ecological races and the nature of species: empirical evidence for the ease of speciation," Philosophical Transactions of the Royal Society B, vol. 363, no. 1506, pp. 2971-2986, 2008.

[108] S. P. Otto and N. H. Barton, "The evolution of recombination: removing the limits to natural selection," Genetics, vol. 147, no. 2, pp. 879-906, 1997.

[109] K. Holsinger, "Pollination biology and the evolution of mating systems in flowering plants," Evolutionary Biology, vol. 29, no. 5, pp. 107-149, 1996.

[110] O. Ronce, I. Olivieri, J. Clobert, and E. Danchin, "Perspective on the study of dispersal evolution," in Dispersal, J. Clobert, E. Danchin, A. Dhondt, and J. Nichols, Eds., pp. 341-357, Oxford University Press, Oxford, UK, 2001. 

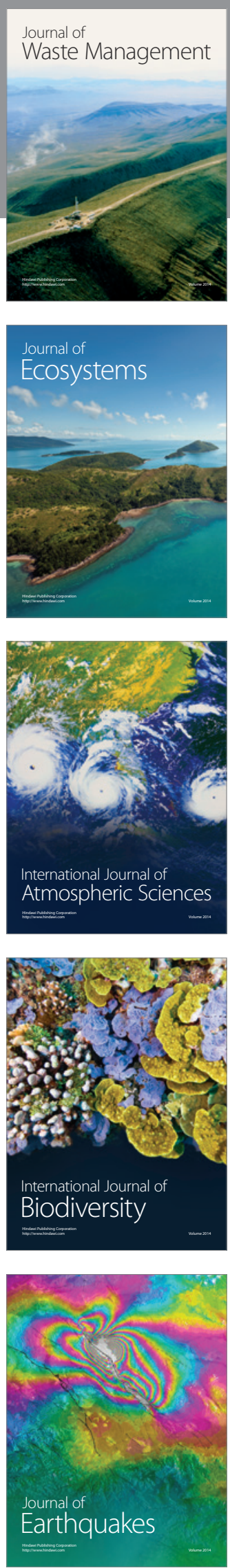
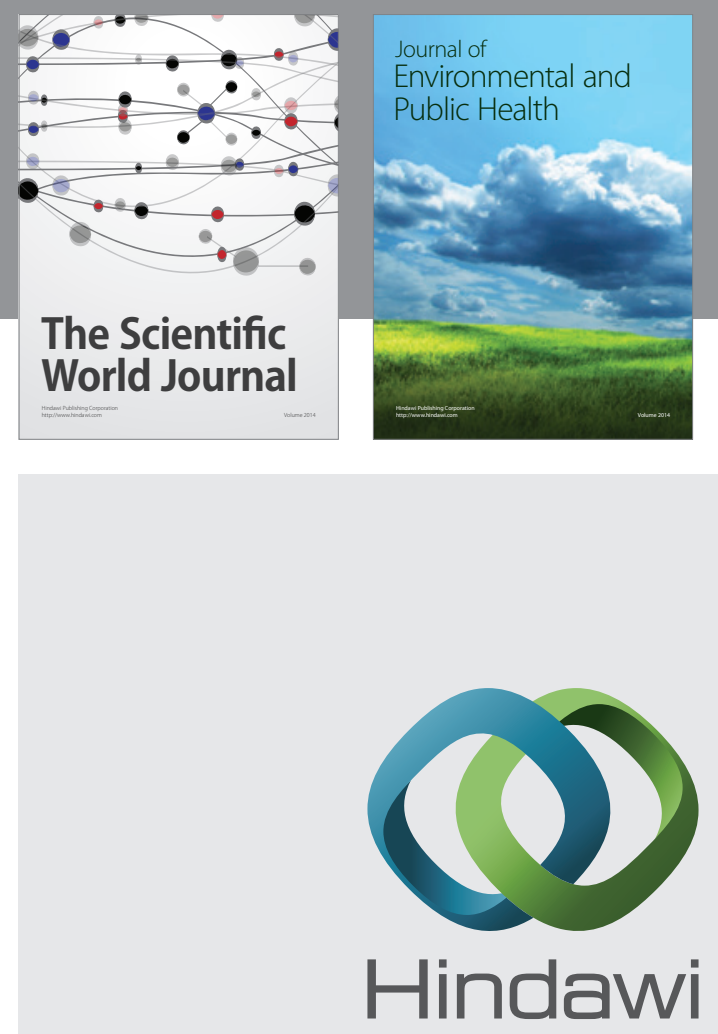

Submit your manuscripts at

http://www.hindawi.com
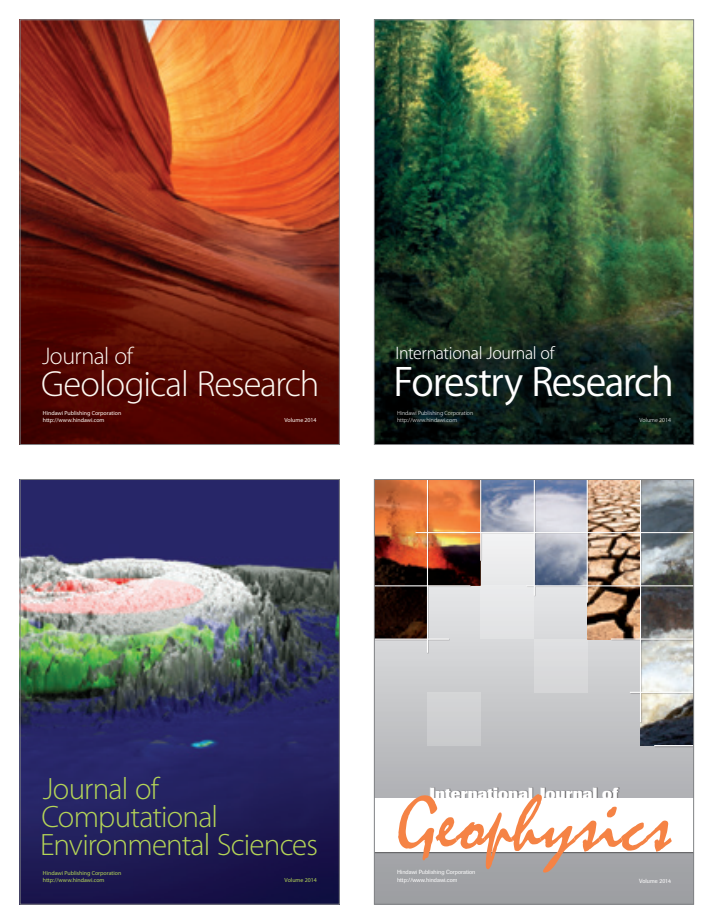
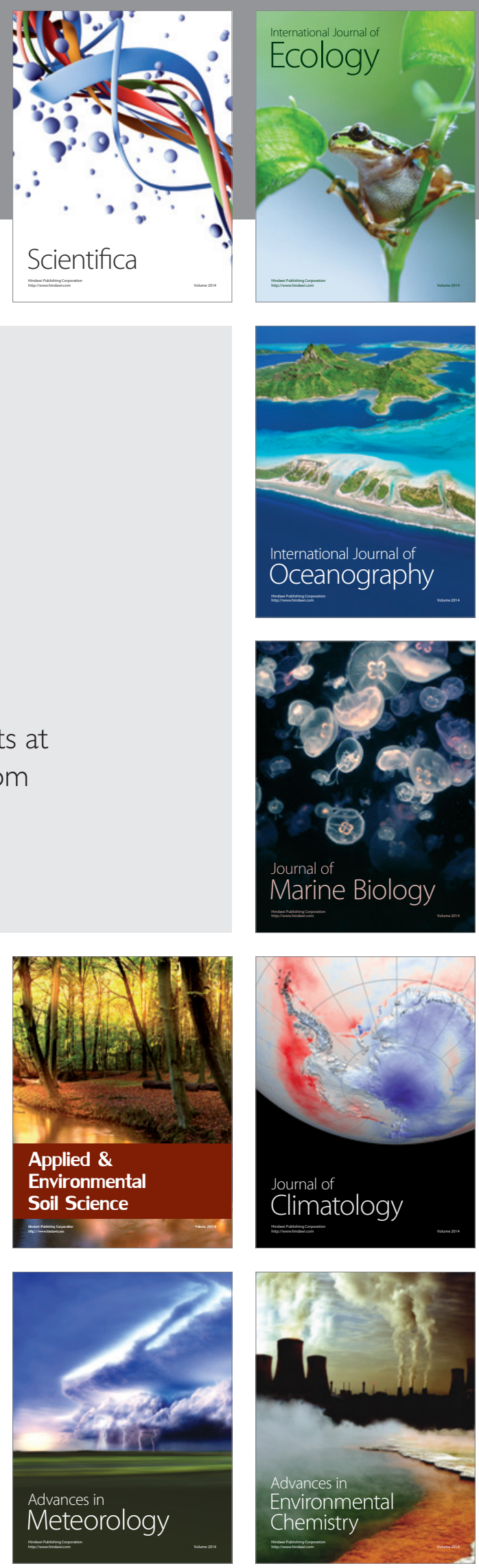